\title{
HAMILTONICITIES OF DOUBLE DOMINATION CRITICAL AND STABLE CLAW-FREE GRAPHS
}

\author{
PaWATON KaEMAWichanuRat \\ Theoretical and Computational Science Center and \\ Department of Mathematics, Faculty of Science \\ King Mongkut's University of Technology Thonburi \\ 126 Pracha Uthit Road, Bang Mod \\ Thung Khru, Bangkok 10140, Thailand \\ e-mail: pawaton.kae@kmutt.ac.th
}

\begin{abstract}
A graph $G$ with the double domination number $\gamma_{\times 2}(G)=k$ is said to be $k$ - $\gamma_{\times 2}$-critical if $\gamma_{\times 2}(G+u v)<k$ for any $u v \notin E(G)$. On the other hand, a graph $G$ with $\gamma_{\times 2}(G)=k$ is said to be $k$ - $\gamma_{\times 2}^{+}$-stable if $\gamma_{\times 2}(G+u v)=k$ for any $u v \notin E(G)$ and is said to be $k-\gamma_{\times 2}^{-}$-stable if $\gamma_{\times 2}(G-u v)=k$ for any $u v \in E(G)$. The problem of interest is to determine whether or not 2-connected $k-\gamma_{\times 2}$-critical graphs are Hamiltonian. In this paper, for $k \geq 4$, we provide a 2 -connected $k-\gamma_{\times 2}$-critical graph which is non-Hamiltonian. We prove that all 2-connected $k-\gamma_{\times 2}$-critical claw-free graphs are Hamiltonian when $2 \leq k \leq 5$. We show that the condition claw-free when $k=4$ is best possible. We further show that every 3 -connected $k-\gamma_{\times 2}$-critical claw-free graph is Hamiltonian when $2 \leq k \leq 7$. We also investigate Hamiltonian properties of $k-\gamma_{\times 2}^{+}$-stable graphs and $k-\gamma_{\times 2}^{-}$-stable graphs.
\end{abstract}

Keywords: double domination, critical, stable, Hamiltonian.

2010 Mathematics Subject Classification: 05C69, 05C45.

\section{REFERENCES}

[1] S. Ao, G. MacGillivray and J. Simmons, Hamiltonian properties of independent domination critical graphs, J. Combin. Math. Combin. Comput. 85 (2013) 107-128.

[2] J. Brousek, Z. Ryjáček and O. Favaron, Forbidden subgraphs, Hamiltonicity and closure in claw-free graphs, Discrete Math. 196 (1999) 29-50. doi:10.1016/S0012-365X(98)00334-3

[3] V. Chvátal, Tough graphs and Hamiltonian circuits, Discrete Math. 306 (2006) 910-917 (reprinted from Discrete Math. 5 (1973) 215-228).

doi:10.1016/j.disc.2006.03.011 
[4] M. Chellali and T.W. Haynes, Double domination stable graphs upon edge removal, Australas. J. Combin. 47 (2010) 157-164.

[5] O. Favaron, F. Tian and L. Zhang, Independence and Hamiltonicity in 3-dominaion critical graphs, J. Graph Theory 25 (1997) 173-184. doi:10.1002/(SICI)1097-0118(199707)25:3〈173::AID-JGT1〉3.0.CO;2-I

[6] E. Flandrin, F. Tian, B. Wei and L. Zhang, Some properties of 3-domination critical graphs, Discrete Math. 205 (1999) 65-76. doi:10.1016/S0012-365X(99)00038-2

[7] Z. Ryjáček, On a closure concept in claw-free graphs, J. Combin. Theory Ser. B 70 (1997) 217-224. doi:10.1006/jctb.1996.1732

[8] P. Kaemawichanurat and L. Caccetta, Hamiltonicity of domination critical claw-free graphs, J. Combin. Math. Combin. Comput. 103 (2017) 39-62.

[9] P. Kaemawichanurat, L. Caccetta and W. Ananchuen, Hamiltonicities of connected domination critical graphs, Ars Combin. 136 (2018) 137-151.

[10] J. Simmons, Closure Operations and Hamitonian Properties of Independent and Total Domination Critical Graphs, Ph.D. Thesis (University of Victoria, 2005).

[11] D.P. Sumner and P. Blitch, Domination critical graphs, J. Combin. Theory Ser. B 34 (1983) 65-76. doi:10.1016/0095-8956(83)90007-2

[12] D.W. Thacker, Double Domination Edge Critical Graph, Master Thesis (East Tennessee State University, 2006).

[13] F. Tian, B. Wei and L. Zhang, Hamiltonicity in 3-domination critical graphs with $\alpha=\delta+2$, Discrete Appl. Math. 92 (1999) 57-70.

doi:10.1016/S0166-218X(98)00149-8

[14] H.C. Wang and L.Y. Kang, Matching properties in double domination edge critical graphs, Discrete Math. Algorithms Appl. 2 (2010) 151-160. doi:10.1142/S1793830910000541

[15] H.C. Wang and E.F. Shan, Some matching properties in $4-\gamma_{\times 2}$-critical graphs, Comput. Math. Appl. 59 (2010) 694-699.

doi:10.1016/j.camwa.2009.10.024

[16] H.C. Wang, E.F. Shan and Y.C. Zhao, 3-factor criticality in double domination edge critical graphs, Graphs Combin. 32 (2016) 1599-1610. doi:10.1007/s00373-015-1670-y

[17] E. Wojcicka, Hamiltonian properties of domination critical graphs, J. Graph Theory 14 (1990) 205-215. doi:10.1002/jgt.3190140209

[18] W. Xiong, H.J. Lai, X. Ma, K. Wang and M. Zhang, Hamilton cycles in 3-connected claw-free and net-free graphs, Discrete Math. 313 (2013) 784-795. doi:10.1016/j.disc.2012.12.016 
[19] Y. Yuansheng, Z. Chengye, L. Xiaohui, J. Yongsong and H. Xin, Some 3-connected 4-edge critical non-Hamiltonian graphs, J. Graph Theory 50 (2005) 316-320. doi:10.1002/jgt.20114

Received 30 October 2017 Revised 7 May 2018

Accepted 7 May 2018 